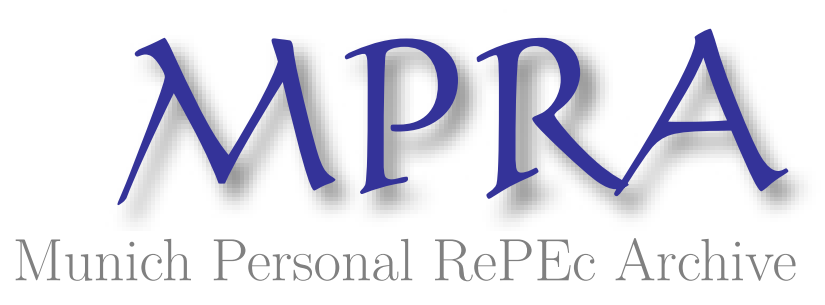

\title{
The Log-GARCH Model via ARMA Representations
}

\author{
Sucarrat, Genaro \\ BI Norwegian Business School
}

30 August 2018

Online at https://mpra.ub.uni-muenchen.de/100386/

MPRA Paper No. 100386, posted 15 May 2020 05:17 UTC 


\title{
The Log-GARCH Model via ARMA Representations*
}

\author{
Genaro Sucarrat ${ }^{\dagger}$
}

This version: 30th August 2018

[First version: 4th December 2017]

\begin{abstract}
The log-GARCH model provides a flexible framework for the modelling of economic uncertainty, financial volatility and other positively valued variables. Its exponential specification ensures fitted volatilities are positive, allows for flexible dynamics, simplifies inference when parameters are equal to zero under the null, and the logtransform makes the model robust to jumps or outliers. An additional advantage is that the model admits ARMA-like representations. This means log-GARCH models can readily be estimated by means of widely available software, and enables a vast range of well-known time-series results and methods. This chapter provides an overview of the log-GARCH model and its ARMA representation(s), and of how estimation can be implemented in practice. After the introduction, we delineate the univariate log-GARCH model with volatility asymmetry ("leverage"), and show how its (nonlinear) ARMA representation is obtained. Next, stationary covariates ("X") are added, before a first-order specification with asymmetry is illustrated empirically. Then we turn our attention to multivariate log-GARCH-X models. We start by presenting the multivariate specification in its general form, but quickly turn our focus to specifications that can be estimated equation-by-equation - even in the presence of Dynamic Conditional Correlations (DCCs) of unknown form. Next, a multivariate non-stationary log-GARCH-X model is formulated, in which the X-covariates can be both stationary and/or nonstationary. A common critique directed towards the log-GARCH model is that its ARCH terms may not exist in the presence of inliers. An own Section is devoted to how this can be handled in practice. Next, the generalisation of log-GARCH models to logarithmic Multiplicative Error Models (MEMs) is made explicit. Finally, the chapter concludes.
\end{abstract}

JEL Classification: C22, C32, C51, C58

Keywords: Financial return, volatility, ARCH, exponential GARCH, log-GARCH, Multivariate GARCH

*I am grateful to the Bilel, a reviewer and Hamdi Raissi for useful comments, suggestions and questions. All errors are mine.

${ }^{\dagger}$ Department of Economics, BI Norwegian Business School, Nydalsveien 37, 0484 Oslo, Norway. Email genaro.sucarrat@bi.no, phone $+47+46410779$, fax $+47+23264788$. Webpage: http://www.sucarrat.net/ 


\section{Contents:}

1 Introduction $\quad 2$

2 Univariate log-GARCH models 5

2.1 The asymmetric $\log -\mathrm{GARCH} \ldots \ldots \ldots . \ldots 5$

2.2 The ARMA representation . . . . . . . . . . . . . . . . 6

2.3 Adding stationary covariates ("X") . . . . . . . . . . . 7

2.4 Estimation of the coefficient covariance matrix . . . . . . . . 7

2.5 Empirical examples . . . . . . . . . . . . . . . . . . 9

3 Multivariate log-GARCH models $\quad 10$

3.1 A multivariate asymmetric log-GARCH-X model . . . . . . . . . 10

3.2 Equation-by-equation estimation . . . . . . . . . . . . . 11

3.3 Non-stationary models . . . . . . . . . . . . . . . . . . . . . 12

3.4 Dynamic Conditional Correlations (DCCs) . . . . . . . . . 15

4 Handling zeros in practice $\quad 15$

5 Modelling positively valued variables $\quad 16$

$\begin{array}{lll}6 & \text { Conclusions } & 17\end{array}$

$\begin{array}{ll}\text { References } & 17\end{array}$

\section{Introduction}

The starting point of Engle's (1982) Autoregressive Conditional Heteroscedasticity (ARCH) class of models is

$$
y_{t}=\mu_{t}+\epsilon_{t}, \quad \epsilon_{t}=\sigma_{t} \eta_{t}, \quad \sigma_{t}>0, \quad \eta_{t} \sim i i d(0,1)
$$

where $y_{t}$ denotes the variable of interest (e.g. financial return), $\mu_{t}$ is the mean specification (e.g. an AR-X model) or simply zero, $\epsilon_{t}$ is the error term or mean-corrected variable of interest, $\sigma_{t}$ is the conditional standard deviation or volatility, and $\eta_{t}$ is an innovation with mean zero and unit variance. Arguably, the most common specification of $\sigma_{t}$ is the first-order Generalised ARCH (GARCH) model of Bollerslev (1986):

$$
\sigma_{t}^{2}=\omega+\alpha_{1} \epsilon_{t-1}^{2}+\beta_{1} \sigma_{t-1}^{2}, \quad \omega>0, \quad \alpha_{1}, \beta_{1} \geq 0 .
$$

Usually, this model is referred to as the GARCH(1,1) model. The log-ARCH class of models was, independently, first proposed by Geweke (1986), Pantula (1986) and Milhøj (1987). However, the idea of modelling the log-variance goes at least back to Park (1966). The logarithmic counterpart of the $\operatorname{GARCH}(1,1)$ is the $\log -\operatorname{GARCH}(1,1)$ model, which is given by

$$
\ln \sigma_{t}^{2}=\omega+\alpha_{1} \ln \epsilon_{t-1}^{2}+\beta_{1} \ln \sigma_{t-1}^{2}, \quad \omega, \alpha_{1}, \beta_{1} \in \mathbb{R} .
$$

Just as in the GARCH model, the $\omega$ is the volatility intercept, $\alpha_{1}$ is the ARCH-parameter and $\beta_{1}$ is the GARCH-parameter: $\omega$ controls the level of volatility (but in a multiplicative 
way), $\alpha_{1}$ controls the impact of shocks or news $\eta_{t-1}^{2}$, whereas $\alpha_{1}+\beta_{1}$ controls the degree to which volatility $\sigma_{t}^{2}$ is persistent. If $\epsilon_{t}$ is a (mean-corrected) daily financial return, then typical estimates of $\alpha_{1}$ and $\beta_{1}$ lie around 0.05 and 0.9 , respectively, both in the $\operatorname{GARCH}(1,1)$ and $\log -\mathrm{GARCH}(1,1)$ cases. See Section 2.5 for an illustration of the latter. Let $\ln ^{+} x=\max \{x, 0\}$. If $\operatorname{Pr}\left(\eta_{t}=0\right)=0, E\left|\ln \eta_{t}^{2}\right|<\infty$ and $E\left(\ln ^{+}\left|\ln \eta_{t}^{2}\right|\right)<\infty$, then a sufficient condition for strict stationarity and ergodicity of (2) is

$$
\left|\alpha_{1}+\beta_{1}\right|<1
$$

see Francq et al. (2013, Theorem 2.1 on p. 36).

From a user-perspective, the log-GARCH model has many attractive features:

- Fitted volatility is guaranteed to be positive due to the exponential specification. This is particularly important in higher order specifications, which may be needed in daily data with weekly periodicity (e.g. electricity prices), and in quarterly and monthly data. For example, when Engle (1982) proposed his ARCH model, he had to substantially restrict his $\mathrm{ARCH}(4)$ specification of quarterly UK inflation uncertainty to ensure positivity of his conditional variance estimates: The specification was assumed to follow $\sigma_{t}^{2}=\omega+\alpha_{1}\left(0.4 \epsilon_{t-1}^{2}+0.3 \epsilon_{t-2}^{2}+0.2 \epsilon_{t-3}^{2}+0.1 \epsilon_{t-4}^{2}\right)$ so that only $\omega$ and $\alpha_{1}$ were estimated, see Engle (1982, p. 1002).

- No non-negativity constraints on parameters. In the GARCH model, parameters must satisfy non-negativity constraints. In the $\operatorname{GARCH}(1,1)$, for example, the constraints are $\omega>0$ and $\alpha_{1}, \beta_{1} \geq 0$. The first means $\sigma_{t}^{2}$ is bounded from below and hence cannot be smaller than $\omega$. The second, i.e. $\alpha_{1}, \beta_{1} \geq 0$, implies that autocorrelations of $\epsilon_{t}^{2}$ are non-negative. The log-GARCH, by contrast, does not impose non-negativity constraints on the parameters. This means its volatility is bounded from below by 0 (and not $\omega$ as in the GARCH), and that it admits negative autocorrelations on $\epsilon_{t}^{2}$. This latter is useful in regular data (e.g. daily data with a weekly 5-day or 7-day cycle, and monthly and quarterly data), since there it is likely that one or more autocorrelations of $\epsilon_{t}^{2}$ are negative, see e.g. Pretis et al. (2018, Section $5.4)$.

- Standard inference valid under nullity of parameters. Often it is of interest to formulate a test in which one or more parameters are 0 under the null, say, $H_{0}: \beta_{1}=0$. A test with this null can be carried out in a standard way (e.g. with a $t$-test) in the log-GARCH model, since the value of $\beta_{1}$ lies in the interior of the admissible parameter space under the null. In the GARCH model, by contrast, $\beta_{1}=0$ lies on the boundary of the admissible parameter space because of the non-negativity constraints. This means standard inference procedures are not valid. The practical implication of this is that it is usually easier to carry out hypothesis tests in the logGARCH model when parameters are 0 under the null. Indeed, such tests can readily be carried out in standard software via the estimated ARMA representations.

- No non-negativity constraints on covariates. Often it is of interest to include covariates in the $\sigma_{t}^{2}$ specification. For example, does a large return yesterday in a different market (say, $x_{t-1}^{2}$ ) increase volatility $\sigma_{t}^{2}$ today? Numerous studies have been undertaken with covariates of this or a similar kind. For brevity, the inclusion 
of covariates is sometimes indicated by "X". In the log-GARCH-X model the covariates are not restricted to be non-negative. In the GARCH-X model, by contrast, the covariates must be non-negative to ensure that $\sigma_{t}^{2}$ is positive, see e.g. Francq and Thieu (2018). This limits the type of questions that can be answered within a GARCH-X model, and compounds the problem described above of inference under nullity of parameters. These restrictions and challenges do not characterise the $\log -\mathrm{GARCH}$.

- Invariance to power-transformations. Consider the $\delta$ th. power $\log$-GARCH $(1,1)$ specification

$$
\ln \sigma_{t}^{\delta}=\omega_{\delta}+\alpha_{1} \ln \left|\epsilon_{t-1}\right|^{\delta}+\beta_{1} \ln \sigma_{t-1}^{\delta}, \quad \delta>0 .
$$

This specification is of interest if the objective is to forecast, say, the conditional standard deviation $\sigma_{t}$ (or any $\sigma_{t}^{\delta}$ with $\delta>0$ ) rather than $\sigma_{t}^{2}$. In contrast to the power GARCH counterpart, (4) can be re-written in terms of its 2 nd. power as

$$
\ln \sigma_{t}^{2}=\omega+\alpha_{1} \ln \epsilon_{t-1}^{2}+\beta_{1} \ln \sigma_{t-1}^{2} \quad \text { with } \quad \omega=\frac{\omega_{\delta}}{\delta} .
$$

In other words, an estimate of (4) for any power $\delta>0$ is straightforwardly obtained via the estimate of (5). The power GARCH model, by contrast, is not characterised by this invariance to power transformations.

- Robustness to outliers. It is well-known that the GARCH model is fragile when outliers or large jumps in $\epsilon_{t}$ are present, see e.g. Carnero et al. (2007) and the references therein. Similarly, the unconditional variance in Nelson's (1991) EGARCH may not exist if $\eta_{t}$ is too fat-tailed, e.g. student's $t$, see Nelson's own discussion in the Appendix (same place). In the log-GARCH, by contrast, the effect of $\eta_{t}^{2}$ is dampened due to the log-transformation. This means the log-GARCH is much more robust to outliers or jumps and fat-tailedness of $\eta_{t}$. This can be illustrated by revisiting the daily Apple log-return series used by Harvey and Sucarrat (2014, pp. 320-321) to illustrate a similar robustness for the Beta-t-EGARCH model. On Thursday 28 September 2000 the firm Apple issued a profit warning after closing hours, which led its stock-value to fall from USD 26.75 to USD 12.88. Volatility, however, was not affected on the subsequent days. Figure 1 contains a snapshot of the event and the surrounding days. The figure plots absolute returns, the fitted conditional standard deviations of a $\operatorname{GARCH}(1,1)$ specification, and the fitted conditional standard deviations of a log-GARCH(1,1). The GARCH forecasts (one-step-ahead) of standard deviations exceed absolute returns for almost two months after the event, a clear-cut example of forecast failure. The forecasts of the log-GARCH, by contrast, remain in the same range of variation as the absolute returns due to the log-transformation. ${ }^{1}$ This provides an empirical example of the GARCH model being prone to forecast failure in the presence of large outliers or jumps.

- Generality of specification. Two common alternatives to the log-GARCH are the Stochastic Volatility (SV) class of models, and the EGARCH of Nelson (1991). In

\footnotetext{
${ }^{1}$ Estimation in R (R Core Team (2018)). The GARCH model is estimated with the garch function from the tseries package of Trapletti and Hornik (2016). The log-GARCH model is estimated via its ARMA representation with the lgarch function from the lgarch package of Sucarrat (2015).
} 
a sense, the log-GARCH is more general than these model-classes, since both admit a log-GARCH representation (but not necessarily vice-versa), see Asai (1998), and Francq et al. (2017).

- Log-GARCH models admit ARMA representations. This was already noted by Pantula (1986), and has been exploited in numerous subsequent works, see e.g. Psaradakis and Tzavalis (1999). The usefulness of this is that a vast number of results, methods and techniques from the time-series literature is available. In particular, widely available software provide routines for the estimation of ARMA and/or VARMA models can be applied, which means univariate and multivariate log-GARCH models can readily be estimated in practice via their (V)ARMA representation(s).

The focus of this chapter is the last of these features. In the next part, Section 2 , we provide an overview of univariate models. We start by outlining an asymmetric specification, before we turn to its ARMA representation. Next we add stochastic conditioning covariates ("X"), and then sketch how estimates of the coefficient-covariances can be obtained in numerical software. We complete the Section by empirical illustrations of the log-GARCH $(1,1)$ model. Section 3 provides an overview of multivariate models. Again, we start by outlining the asymmetric specification and its corresponding VARMA and VARMA-X representations. Next, we turn to specifications that are amenable to equation-by-equation estimation, both stationary and non-stationary versions, even in the presence of Dynamic Conditional Correlations (DCCs) of unknown form. The focus on multivariate specifications that can be estimated equation-by-equation is motivated by the fact estimation becomes infeasible in practice as the dimension grows too large. We end the section with a short note on how models of Dynamic Conditional Correlations (DCCs) can be estimated subsequently. Section 4 provides some suggestions on how to handle zeros in practice, whereas Section 5 outlines how log-GARCH models can be used to model positively valued variables. Finally, Section 6 concludes and provides suggestions for further research.

\section{Univariate log-GARCH models}

\subsection{The asymmetric log-GARCH}

Financial returns are often more volatile after a fall in price compared to a rise. This is usually referred to as asymmetry or leverage. To accommodate this commonly found feature, Francq et al. (2013) proposed the asymmetric log-GARCH. If $\operatorname{Pr}\left(\eta_{t}=0\right)=0$ for all $t$, then their asymmetric log-GARCH can be re-parametrised as

$$
\ln \sigma_{t}^{2}=\omega+\sum_{i=1}^{p} \alpha_{i} \ln \epsilon_{t-i}^{2}+\sum_{j=1}^{q} \beta_{j} \ln \sigma_{t-j}^{2}+\sum_{k=1}^{r} \gamma_{k} 1_{\left\{\epsilon_{t-k}<0\right\}} \ln \epsilon_{t-k}^{2}
$$

where

$$
1_{\left\{\epsilon_{t}<0\right\}} \ln \epsilon_{t}^{2}= \begin{cases}\ln \epsilon_{t}^{2} & \text { if } \epsilon_{t}<0 \\ 0 & \text { if } \epsilon_{t}<0\end{cases}
$$

is the asymmetry or leverage term. The advantages of the re-parametrisation in (6) are that it is more straightforward to test for the presence of asymmetry in practice, 
and that it closely resembles the most common asymmetric non-exponential GARCHcounterpart of Glosten et al. (1993). In the $\log -\mathrm{GARCH}(1,1)$, for example, asymmetry can be tested by means of a simple $t$-test. The re-parametrisation implies, however, that the sufficient conditions for strict stationarity and ergodicity (i.e. Theorem 2.1 in Francq et al. (2013, p. 36)) also needs to be re-parametrised. For example, in the first order case (i.e. $p=q=r=1$ ), the sufficient condition becomes

$$
\left|\alpha_{1}+\beta_{1}\right|^{\operatorname{Pr}\left(\eta_{t}>0\right)} \cdot\left|\alpha_{1}+\beta_{1}+\gamma_{1}\right|^{1-\operatorname{Pr}\left(\eta_{t}>0\right)}<1 .
$$

In the absence of asymmetry we obtain the usual condition in (3), i.e. $\left|\alpha_{1}+\beta_{1}\right|<1$.

\subsection{The ARMA representation}

If $\operatorname{Pr}\left(\eta_{t}=0\right)=0$ and $E\left|\ln \eta_{t}^{2}\right|<\infty$, then (6) admits, almost surely, a (nonlinear in variables) $\operatorname{ARMA}(p, q)$ representation. It is obtained in two steps. First, $\ln \eta_{t}^{2}$ is added to each side of (6). Second, $\sum_{j=1}^{q} \beta_{j}\left(\ln \eta_{t}^{2}-E\left(\ln \eta_{t}^{2}\right)\right)-\sum_{j=1}^{q} \beta_{j}\left(\ln \eta_{t}^{2}-E\left(\ln \eta_{t}^{2}\right)\right)$ is added to the right-hand side. Re-organising gives the nonlinear ARMA representation

$$
\ln \epsilon_{t}^{2}=\omega^{*}+\sum_{i=1}^{p} \phi_{i} \ln \epsilon_{t-i}^{2}+\sum_{j=1}^{q} \theta_{j} u_{t-j}^{2}+\sum_{k=1}^{r} \gamma_{k} 1_{\left\{\epsilon_{t-k}<0\right\}} \ln \epsilon_{t-k}^{2}+u_{t}, \quad u_{t} \sim i i d\left(0, \sigma_{u}^{2}\right)
$$

where

$$
\omega^{*}=\omega+\left(1-\sum_{j=1}^{q} \beta_{j}\right) \cdot E\left(\ln \eta_{t}^{2}\right), \quad \phi_{i}=\alpha_{i}+\beta_{i}, \quad \theta_{j}=-\beta_{j}, \quad u_{t}=\ln \eta_{t}^{2}-E\left(\ln \eta_{t}^{2}\right)
$$

If, in addition, $E\left[\left(\ln \eta_{t}^{2}\right)^{2}\right]<\infty$, then $\sigma_{u}^{2}<\infty$ with $\sigma_{u}^{2}=E\left[\left(\ln \eta_{t}^{2}\right)^{2}\right]-E\left(\ln \eta_{t}^{2}\right)^{2}$. Note that the specification is a nonlinear (in variables) ARMA due to the asymmetry terms. The stationarity conditions of Francq et al. (2013) still apply, since $\ln \epsilon_{t}^{2}$ is simply a sum of the stationary variables $\ln \sigma_{t}^{2}$ and $\ln \eta_{t}^{2}$. The model is therefore amenable to estimation by well-known ARMA-methods and widely available software. All the ARCH and GARCH parameters are identified via the relations in (8), and inference - even under the null of zero parameters - is readily carried out via a suitable transformation of the estimated coefficient covariance matrix, see Section 2.4. However, to identify the volatility intercept $\omega$ an estimate of $E\left(\ln \eta_{t}^{2}\right)$ is needed, and $E\left(\ln \eta_{t}^{2}\right)$ depends on the distribution of $\eta_{t}^{2}$. Sucarrat et al. (2016) show that, under mild and general assumptions,

$$
-\ln \left[\frac{1}{T} \sum_{t=1}^{T} \exp \left(\widehat{u}_{t}\right)\right] \underset{p}{\longrightarrow} E\left(\ln \eta_{t}^{2}\right)
$$

where $T$ is the sample size and $\widehat{u}_{t}$ is the residual from the estimated ARMA representation. Note that the expression inside the square brackets of (9) is the smearing estimator of Duan (1983). The motivation behind this estimator is that, if $E\left(\eta_{t}^{2}\right)=1$ and $E\left(\ln \eta_{t}^{2}\right)<$ $\infty$, then the population counterpart is equal to $E\left(\ln \eta_{t}^{2}\right)$ :

$$
-\ln E\left[e^{u_{t}}\right]=-\ln E\left[e^{\ln \eta_{t}^{2}-E\left(\ln \eta_{t}^{2}\right)}\right]=-\ln \left[\frac{1}{e^{E\left(\ln \eta_{t}^{2}\right)}} \cdot E\left(\eta_{t}^{2}\right)\right]=E\left(\ln \eta_{t}^{2}\right)
$$


Subject to suitable assumptions, therefore, consistent estimation of the ARMA representation $(7)$ and the $\log$-moment $E\left(\ln \eta_{t}^{2}\right)$, leads to consistent estimation of all the log-GARCH parameters in (6).

Another notable property of the estimator in (9) is that it ensures the sample variance of the standardised residuals $\left\{\widehat{\eta}_{t}\right\}=\left\{\epsilon_{t} / \widehat{\sigma}_{t}\right\}$, where $\widehat{\sigma}_{t}^{2}$ is the fitted value of $\sigma_{t}^{2}$, is approximately equal to 1 in empirical applications. This is required for $\widehat{\sigma}_{t}^{2}$ to be a valid estimate of the conditional variance $\sigma_{t}^{2}$. To see that the estimator in (9) ensures that the sample variance of $\left\{\widehat{\eta}_{t}\right\}$ is approximately equal to 1 , let $\widehat{\eta}_{t}^{*}=\epsilon_{t} / \widehat{\sigma}_{t}^{*}$ denote the residual scaled by the square root of the fitted value of the exponentiated ARMA-representation: $\widehat{\sigma}_{t}^{* 2}=\exp \left(\widehat{\ln \epsilon_{t}^{2}}\right)$, where $\widehat{\ln \epsilon_{t}^{2}}$ is the fitted value of the ARMA-representation. Noting that we also have $\widehat{\eta}_{t}^{*}=\exp \left(\widehat{u}_{t} / 2\right)$, it follows that

$$
\frac{\widehat{\eta}_{t}^{*}}{\sqrt{T^{-1} \sum_{t=1}^{T} \exp \left(\widehat{u}_{t}\right)}}=\frac{\widehat{\eta}_{t}^{*}}{\exp \left(\ln T^{-1} \sum_{t=1}^{T} \exp \left(\widehat{u}_{t}\right) / 2\right)}=\frac{\epsilon_{t}}{\exp \left(\widehat{\ln \epsilon_{t}^{2}} / 2-\widehat{E}\left(\ln \eta_{t}^{2}\right) / 2\right)}=\widehat{\eta}_{t}
$$

where $\widehat{E}\left(\ln \eta_{t}^{2}\right)$ is the estimator in (9). In other words, the smearing estimate $T^{-1} \sum_{t=1}^{T} \exp \left(\widehat{u}_{t}\right)$ is approximately equal to the sample variance of $\left\{\widehat{\eta}_{t}^{*}\right\}$, thus ensuring the sample variance of $\left\{\widehat{\eta}_{t}\right\}$ is always approximately equal to 1 in empirical applications.

\subsection{Adding stationary covariates ("X")}

Let $\boldsymbol{x}_{t}=\left(x_{1 t}, \ldots, x_{s t}\right)^{\prime}$ denote a vector of strictly stationary and ergodic covariates. The (asymmetric) log-GARCH-X model is given by

$$
\ln \sigma_{t}^{2}=\omega+\sum_{i=1}^{p} \alpha_{i} \ln \epsilon_{t-i}^{2}+\sum_{j=1}^{q} \beta_{j} \ln \sigma_{t-j}^{2}+\sum_{k=1}^{r} \gamma_{k} 1_{\left\{\epsilon_{t-k}<0\right\}} \ln \epsilon_{t-k}^{2}+\sum_{l=1}^{s} \lambda_{l} x_{l, t-1} .
$$

A common example of a covariate is realised volatility, i.e. a volatility proxy, but another example is extended asymmetry. In other words, the extended asymmetric log-GARCH model of Francq et al. (2017) is nested in (10). The (nonlinear) ARMA-X representation is obtained in the same way as earlier (see above), and it is given by

$$
\ln \epsilon_{t}^{2}=\omega^{*}+\sum_{i=1}^{p} \phi_{i} \ln \epsilon_{t-i}^{2}+\sum_{j=1}^{q} \theta_{j} u_{t-j}^{2}+\sum_{k=1}^{r} \gamma_{k} 1_{\left\{\epsilon_{t-k}<0\right\}} \ln \epsilon_{t-k}^{2}+\sum_{l=1}^{s} \lambda_{l} x_{l, t-1}+u_{t},
$$

where the relations between the log-GARCH and ARMA parameters are exactly as before, i.e. they are given by (8). Also, as noted earlier, no non-negativity constraints on the parameters $\left(\lambda_{1}, \ldots, \lambda_{s}\right)^{\prime}$ nor on the covariates $\boldsymbol{x}_{t}$ are needed. Accordingly, standard inference methods are available under the null of $0 \mathrm{~s}$ on one or more of the $\lambda_{1}, \ldots, \lambda_{s}$, i.e. that one or more covariate has no impact on volatility. To estimate $E\left(\ln \eta_{t}^{2}\right)$, the same formula as earlier, i.e. (9), can be used. Estimation of (10), therefore, can straightforwardly be undertaken in widely available software.

\subsection{Estimation of the coefficient covariance matrix}

For inference on the parameters an estimate of the coefficient covariance matrix is needed, and this expression depends on the estimator. The two most common estimators of 
ARMA-models are Least Squares (LS) and Gaussian Maximum Likelihood (ML). Both provide consistent and asymptotically normal estimates under mild assumptions - even when the error $u_{t}$ is non-Gaussian, and most of the asymptotic properties of the two estimators are identical, see e.g. Brockwell and Davis (2006). The LS and Gaussian ML estimators are asymptotically efficient when $\eta_{t}$ is sufficiently fat-tailed or skewed (or both). If, however, $\eta_{t}$ is Gaussian, then improved efficiency can be achieved with the exponential Chi-squared (Quasi) ML estimator proposed by Francq and Sucarrat (2018). Here, we outline the details of the LS estimator, but the approach is similar for both the Gaussian and Chi-squared ML estimators.

Let $\boldsymbol{\varphi}=\left(\omega^{*}, \phi_{1}, \ldots, \phi_{p}, \theta_{1}, \ldots, \theta_{q}, \gamma_{1}, \ldots, \gamma_{r}, \lambda_{1}, \ldots, \lambda_{s}\right)^{\prime}$ denote the parameter of the ARMA representation given by (11), and let

$$
\widehat{\varphi}=\arg \min _{\varphi} \frac{1}{T} \sum_{t=1}^{T} u_{t}^{2}
$$

denote its Least Squares (LS) estimate. Often, numerical software provide utility functions for the computation of the Hessian at the optimum. Francq and Sucarrat (2017, pp. 2728) show that this can be used to build an estimate of the coefficient covariance matrix. Specifically, they show that an estimate of the asymptotic coefficient matrix is obtained as

$$
\left(\frac{1}{T} \sum_{t=1}^{T} \widehat{u}_{t}^{2}\right) \cdot 2 \cdot \widehat{\boldsymbol{S}}^{-1}
$$

where $\widehat{u}_{t}$ is the residual of the estimated ARMA-representation and $\widehat{\boldsymbol{S}}$ is the Hessian at $\widehat{\varphi}$ based on (12). If LS estimation is implemented by minimising the sum instead of the average, i.e.

$$
\widehat{\boldsymbol{\varphi}}=\arg \min _{\varphi} \sum_{t=1}^{T} u_{t}^{2},
$$

then the estimate of the asymptotic coefficient matrix is modified to

$$
\left(\frac{1}{T} \sum_{t=1}^{T} \widehat{u}_{t}^{2}\right) \cdot 2 T \cdot \widehat{\boldsymbol{S}}^{-1},
$$

where $\widehat{\boldsymbol{S}}$ is now the Hessian at $\widehat{\boldsymbol{\varphi}}$ based on (13).

Let $\boldsymbol{\zeta}=\left(\omega, \alpha_{1}, \ldots, \alpha_{p}, \beta_{1}, \ldots, \beta_{q}, \gamma_{1}, \ldots, \gamma_{r}, \lambda_{1}, \ldots, \lambda_{s}\right)^{\prime}$ denote the parameter of the $\log$-GARCH specification (10), and let $\widehat{\zeta}$ denote its estimate. An estimate of its asymptotic coefficient matrix is available by using the relationships between the log-GARCH and ARMA-parameters given by (8). For example, if $\widehat{\operatorname{Var}}(\widehat{x})$ and $\widehat{\operatorname{Cov}}(\widehat{x}, \widehat{y})$ denote the variance of the estimate $\widehat{x}$ and the covariance of the estimates $\widehat{x}$ and $\widehat{y}$, respectively, then the variance of the ARCH-parameter $\widehat{\alpha}_{i}$ is obtained as $\widehat{\operatorname{Var}}\left(\widehat{\alpha}_{i}\right)=\widehat{\operatorname{Var}}\left(\widehat{\phi}_{i}\right)+\widehat{\operatorname{Var}}\left(\widehat{\theta}_{i}\right)+2 \widehat{\operatorname{Cov}}\left(\widehat{\phi}_{i}, \widehat{\theta}_{i}\right)$. Similarly, the variance of the GARCH-parameter $\widehat{\beta}_{i}$ is obtained as $\widehat{\operatorname{Var}}\left(\widehat{\beta}_{i}\right)=\widehat{\operatorname{Var}}\left(-\widehat{\theta}_{i}\right)=$ $\widehat{\operatorname{Var}}\left(\widehat{\theta}_{i}\right)$. All the variances and covariances are readily available in this way, apart from those associated with the estimate of the log-GARCH intercept $\widehat{\omega}$. These computations are more involved and requires the use of the delta-method, see Francq and Sucarrat (2017, pp. 21-22). 


\subsection{Empirical examples}

To provide an empirical illustration of the log-GARCH model, we re-visit six daily financial return series: The FTSE100 and SP100 indices (source: Bloomberg), the Apple stock price (source: Yahoo Finance, https://yahoo.finance.com), the USD/EUR exchange rate (source: The European Central Bank, http://www.ecb.int/), the brent blend oilprice (source: The US Energy Information Agency, http://www.eia.gov/) and the gold price (source: Kitco, http://www.kitco.com/). The first two return series were studied in Francq and Sucarrat (2017, Section 5.1), whereas the latter four return series were studied in Harvey and Sucarrat (2014, Section 6). Note that the Apple series is the same as the one used to illustrate the robustness to outliers of log-GARCH models in the introduction (Section 1).

Let $P_{t}$ denote the index-value or price of the asset in question in day $t$. The return $y_{t}=\epsilon_{t}$ is computed as the log-return in percent, i.e. $\epsilon_{t}=\left(\ln P_{t}-\ln P_{t-1}\right) \cdot 100$. The sample periods and descriptive statistics of the returns are contained in the upper part of Table 1, whereas Figure 2 contains graphs of the return series. As commonly found, the returns exhibit excess kurtosis relative to the normal distribution, and first-order ARCH at 5\% and higher significance levels according to a Ljung and Box (1979) test for first-order autocorrelation in $\epsilon_{t}^{2}$. Also, the plots in Figure 2 confirm that volatility is persistent in the sense that the returns are characterised by volatility clustering.

Arguably, the most common volatility model is the plain $\operatorname{GARCH}(1,1)$. The plain $\log -\mathrm{GARCH}(1,1)$ counterpart is given by

$$
\ln \sigma_{t}^{2}=\omega+\alpha_{1} \ln \epsilon_{t-1}^{2}+\beta_{1} \ln \sigma_{t-1}^{2},
$$

and estimates of this model are contained in the middle part of Table 1. Estimation is undertaken via the $\operatorname{ARMA}(1,1)$ representation with the lgarch function from the $R$ package lgarch, see Sucarrat (2015). Usually, in ordinary $\operatorname{GARCH}(1,1)$ models, the estimate of the ARCH parameter $\alpha_{1}$ lies around 0.05 , and the estimate of the GARCH parameter $\beta_{1}$ lies around 0.95 . The results show that this is also the case for the logGARCH $(1,1)$ models. When estimation is via the ARMA-representation, then an estimate of $E\left(\ln \eta_{t}^{2}\right)$ is needed in order to estimate $\omega$. If $\eta_{t} \sim N(0,1)$, then $E\left(\ln \eta_{t}^{2}\right)=-1.27$. In other words, the discrepancy from -1.27 can be viewed as a measure of departure from normality. For example, if $\eta_{t}$ is a standardised $t$ with 10 degrees of freedom (a "moderate" departure from normality), then $E\left(\ln \eta_{t}^{2}\right)=-1.39$. The estimates of $E\left(\ln \eta_{t}^{2}\right)$ range from -1.375 to -1.522 , which suggests $\eta_{t}$ is non-normal, albeit not dramatically so.

Often daily financial return series exhibit volatility asymmetry, i.e. a negative return tends to increase the volatility on the subsequent day. For stocks, this is typically referred to as a leverage effect, since leverage is often cited as the reason for the effect. For other return series, the more generic label "asymmetry" may be more appropriate, since the effect can be positive instead of negative, and since the reason for asymmetry may not be leverage. For exchange rates, for example, the presence and sign of asymmetry will usually depend on the relative strength of the two currencies in question. In other words, asymmetry is unlikely to be present in the USD/EUR exchange rate, since both the USD and Euro currencies are considered as strong currencies in international money markets. To explore the presence of volatility asymmetry in the six return series we fit a logGARCH(1,1) model with extended asymmetry, i.e.

$$
\ln \sigma_{t}^{2}=\omega+\alpha_{1} \ln \epsilon_{t-1}^{2}+\beta_{1} \ln \sigma_{t-1}^{2}+\gamma_{1} 1_{\left\{\epsilon_{t-1}<0\right\}} \ln \epsilon_{t-1}^{2}+\lambda_{1} 1_{\left\{\epsilon_{t-1}<0\right\}} .
$$


The $1_{\left\{\epsilon_{t-1}<0\right\}} \ln \epsilon_{t-1}^{2}$ is the ordinary asymmetry term, and $1_{\left\{\epsilon_{t-1}<0\right\}}$ is the extended asymmetry term. As noted by Francq et al. (2017), to ensure invariance to scale-transformations the extended asymmetry term is needed when the ordinary asymmetry term is present. If we use \pm 2 as critical values in a two-sided $t$-test with zero as null, then both the ordinary and extended asymmetry terms are significant for the stock returns (i.e. FTSE100, SP100 and Apple). For the remaining returns, however, neither the ordinary nor the extended term is significant. In other words, the results suggest the stock returns tend to be more volatile on days subsequent to a negative return, but not the exchange rate, oilprice nor the gold return.

\section{Multivariate log-GARCH models}

Let $\boldsymbol{y}_{t}=\left(y_{1 t}, \ldots, y_{M t}\right)^{\prime}$ denote an $M$-dimensional vector of variables (e.g. financial returns) at $t$. A generic model of $\boldsymbol{y}_{t}$ can be written as (see e.g. Engle (2002))

$$
\begin{aligned}
\boldsymbol{y}_{t} & =\boldsymbol{\mu}_{t}+\boldsymbol{\epsilon}_{t}, \\
\boldsymbol{\epsilon}_{t} & =\left(\epsilon_{1 t}, \ldots, \epsilon_{M t}\right)^{\prime}, \quad \boldsymbol{H}_{t}=E_{t-1}\left(\boldsymbol{\epsilon}_{t} \boldsymbol{\epsilon}_{t}^{\prime}\right), \quad \boldsymbol{D}_{t}^{2}=\operatorname{diag}\left(\boldsymbol{H}_{t}\right), \\
\boldsymbol{\eta}_{t} & =\boldsymbol{D}_{t}^{-1} \boldsymbol{\epsilon}_{t}, \quad \boldsymbol{R}_{t}=E_{t-1}\left(\boldsymbol{\eta}_{t} \boldsymbol{\eta}_{t}^{\prime}\right)
\end{aligned}
$$

where $\boldsymbol{\mu}_{t}$ is, say, a VARMA-X model, $\boldsymbol{\epsilon}_{t}=\left(\epsilon_{1 t}, \ldots, \epsilon_{M t}\right)^{\prime}$ is the error term, $\boldsymbol{H}_{t}$ is an $M \times M$ covariance matrix conditional on the past information $\mathcal{F}_{t-1}, E_{t-1}(\cdot)$ is shorthand notation for $E\left(\cdot \mid \mathcal{F}_{t-1}\right), \boldsymbol{D}_{t}^{2}$ is a diagonal $M \times M$ matrix with the conditional variance or volatility $\boldsymbol{\sigma}_{t}^{2}=\left(\sigma_{1 t}^{2}, \ldots, \sigma_{M t}^{2}\right)^{\prime}$ on the diagonal, $\boldsymbol{\eta}_{t}=\left(\eta_{1 t}, \ldots, \eta_{M t}\right)^{\prime}$ is the standardised error, i.e. $E\left(\boldsymbol{\eta}_{t}\right)=\mathbf{0}$ and $\operatorname{Var}\left(\boldsymbol{\eta}_{t}\right)=\mathbf{1}$ where $\mathbf{0}$ and $\mathbf{1}$ are $M \times 1$ vectors, $\boldsymbol{D}_{t}^{-1}$ is a diagonal $M \times M$ matrix with $\left(1 / \sigma_{1 t}, \ldots, 1 / \sigma_{M t}\right)^{\prime}$ on the diagonal and $\boldsymbol{R}_{t}$ is the correlation matrix conditional on the past. The relationships between $\boldsymbol{H}_{t}$ and $\boldsymbol{R}_{t}$ are given by $\boldsymbol{H}_{t}=\boldsymbol{D}_{t} \boldsymbol{R}_{t} \boldsymbol{D}_{t}$ and $\boldsymbol{R}_{t}=\boldsymbol{D}_{t}^{-1} \boldsymbol{H}_{t} \boldsymbol{D}_{t}^{-1}$.

\subsection{A multivariate asymmetric log-GARCH-X model}

The multivariate asymmetric log-GARCH-X model is given by

$$
\ln \boldsymbol{\sigma}_{t}^{2}=\boldsymbol{\omega}+\sum_{i=1}^{p} \boldsymbol{\alpha}_{i} \ln \boldsymbol{\epsilon}_{t-i}^{2}+\sum_{j=1}^{q} \boldsymbol{\beta}_{j} \ln \boldsymbol{\sigma}_{t-j}^{2}+\sum_{k=1}^{r} \boldsymbol{\gamma}_{k} \mathbf{1}_{\left\{\boldsymbol{\epsilon}_{t-k}<0\right\}} \ln \boldsymbol{\epsilon}_{t-k}^{2}+\boldsymbol{\lambda} \boldsymbol{x}_{t-1}
$$

where $\ln \boldsymbol{\sigma}_{t}^{2}=\left(\ln \sigma_{1 t}^{2}, \ldots, \ln \sigma_{M t}^{2}\right)^{\prime}, \boldsymbol{\omega}=\left(\omega_{1}, \ldots, \omega_{M}\right)^{\prime}, \ln \boldsymbol{\epsilon}_{t-i}^{2}=\left(\ln \epsilon_{1, t-i}^{2}, \ldots, \ln \epsilon_{M, t-i}^{2}\right)^{\prime}$, $\mathbf{1}_{\left\{\boldsymbol{\epsilon}_{t-k}<0\right\}} \ln \boldsymbol{\epsilon}_{t-k}^{2}=\left(1_{\left\{\epsilon_{1, t-k}<0\right\}} \ln \epsilon_{1, t-k}^{2}, \ldots, 1_{\left\{\epsilon_{M, t-k}<0\right\}} \ln \epsilon_{M, t-k}^{2}\right)^{\prime}$ and $\boldsymbol{x}_{t-1}=\left(x_{1, t-1}, \ldots, x_{M, t-1}\right)^{\prime}$ are all $M \times 1$ vectors, and where

$$
\begin{gathered}
\boldsymbol{\alpha}_{i}=\left(\begin{array}{ccc}
\alpha_{11 . i} & \cdots & \alpha_{1 M . i} \\
\vdots & \ddots & \vdots \\
\alpha_{M 1 . i} & \cdots & \alpha_{M M . i}
\end{array}\right), \quad \boldsymbol{\beta}_{j}=\left(\begin{array}{ccc}
\beta_{11 . j} & \cdots & \beta_{1 M . j} \\
\vdots & \ddots & \vdots \\
\beta_{M 1 . j} & \cdots & \beta_{M M . j}
\end{array}\right), \\
\gamma_{k}=\left(\begin{array}{ccc}
\gamma_{11 . k} & \cdots & \gamma_{1 M . k} \\
\vdots & \ddots & \vdots \\
\gamma_{M 1 . k} & \cdots & \gamma_{M M . k}
\end{array}\right), \quad \boldsymbol{\lambda}_{l}=\left(\begin{array}{ccc}
\lambda_{11 . l} & \cdots & \lambda_{1 M . l} \\
\vdots & \ddots & \vdots \\
\lambda_{M 1 . l} & \cdots & \lambda_{M M . l}
\end{array}\right)
\end{gathered}
$$


are all $M \times M$ matrices. The (nonlinear) VARMA-X representation is obtained in the same way as in the univariate case, and it is given by

$$
\ln \boldsymbol{\epsilon}_{t}^{2}=\boldsymbol{\omega}^{*}+\sum_{i=1}^{p} \boldsymbol{\phi}_{i} \ln \boldsymbol{\epsilon}_{t-i}^{2}+\sum_{j=1}^{q} \boldsymbol{\theta}_{j} \boldsymbol{u}_{t-j}^{2}+\sum_{k=1}^{r} \boldsymbol{\gamma}_{k} \mathbf{1}_{\left\{\boldsymbol{\epsilon}_{t-k}<0\right\}} \ln \boldsymbol{\epsilon}_{t-k}^{2}+\lambda \boldsymbol{x}_{t-1}+\boldsymbol{u}_{t},
$$

where

$$
\boldsymbol{\omega}^{*}=\boldsymbol{\omega}+\left(\boldsymbol{I}-\sum_{j=1}^{q} \boldsymbol{\beta}_{j}\right) E\left(\ln \boldsymbol{\eta}_{t}^{2}\right), \quad \boldsymbol{\phi}_{i}=\boldsymbol{\alpha}_{i}+\boldsymbol{\beta}_{i}, \quad \boldsymbol{\theta}_{j}=-\boldsymbol{\beta}_{j}, \quad \boldsymbol{u}_{t}=\ln \boldsymbol{\eta}_{t}^{2}-E\left(\ln \boldsymbol{\eta}_{t}^{2}\right) .
$$

Without asymmetry (i.e. $\gamma_{1}=\cdots=\gamma_{r}=0$ ), (17) is simply a VARMA-X model. To conduct inference on the log-GARCH parameters, an approach similar to the one outlined in Section 2.4 can be used.

If $\boldsymbol{\eta}_{t}$ is iid, then the conditional correlation matrix $\boldsymbol{R}_{t}$ is constant, so that (14) is a Constant Conditional Correlation (CCC) model. Under suitable stationarity and regularity conditions, the (nonlinear) VARMA-X representation (17) can then be estimated by common methods, e.g. multivariate Gaussian QML. If $\boldsymbol{R}_{t}$ is time-varying (and stationary), then a reasonable conjecture is that estimates will still be consistent subject to suitable assumptions. However, the asymptotic properties of such an estimator are currently unknown.

\subsection{Equation-by-equation estimation}

Multivariate volatility models are plagued by the "curse of dimensionality": As the dimension grows, estimation becomes infeasible due to the large amount of parameters that are estimated. One solution, if available, is equation-by-equation estimation. For this to be possible the GARCH-matrices (i.e. $\boldsymbol{\beta}_{1}, \ldots, \boldsymbol{\beta}_{q}$ ) must all be diagonal, and

each $\eta_{m t}, m=1, \ldots, M$, must be independent of the past information $\mathcal{F}_{t-1}$,

see Francq and Zakoïan (2016). Francq and Sucarrat (2017) propose a first order version (i.e. $p=q=1$ ) of the multivariate log-GARCH-X that satisfies these properties, and which allows for certain types of Dynamic Conditional Correlations (DCCs) of unknown form. A generalisation of their model allows for higher orders and asymmetry, and the $m$ th. equation in such a generalised model is given by

$$
\ln \sigma_{m t}^{2}=\omega_{m}+\sum_{i=1}^{p} \boldsymbol{\alpha}_{m . i} \ln \boldsymbol{\epsilon}_{t-i}^{2}+\sum_{j=1}^{q} \beta_{m m . j} \ln \sigma_{m, t-j}^{2}+\sum_{k=1}^{r} \boldsymbol{\gamma}_{m . k} \mathbf{1}_{\left\{\boldsymbol{\epsilon}_{t-k}<0\right\}} \ln \boldsymbol{\epsilon}_{t-k}^{2}+\boldsymbol{\lambda}_{m} \boldsymbol{x}_{t-1},
$$

where $\boldsymbol{\alpha}_{m . i}, \boldsymbol{\gamma}_{m . k}$ and $\boldsymbol{\lambda}_{m}$ are $1 \times M$ vectors made up of the $m$ th. row in the matrices $\boldsymbol{\phi}_{i}$, $\gamma_{k}$ and $\boldsymbol{\lambda}$, respectively. The $\beta_{m m . j}$ is the $m$ th. element of the $m$ th. column in the diagonal $\operatorname{matrix} \boldsymbol{\beta}_{j}$.

The univariate ARMA-X representation of the $m$ th. equation is

$$
\begin{aligned}
\ln \epsilon_{m t}^{2}=\omega_{m}^{*}+\sum_{i=1}^{p} & \phi_{m . i} \ln \boldsymbol{\epsilon}_{t-i}^{2}+\sum_{j=1}^{q} \theta_{m m . j} u_{m, t-j} \\
& +\sum_{k=1}^{r} \boldsymbol{\gamma}_{m . k} \mathbf{1}_{\left\{\boldsymbol{\epsilon}_{t-k}<0\right\}} \ln \boldsymbol{\epsilon}_{t-k}^{2}+\boldsymbol{\lambda}_{m} \boldsymbol{x}_{t-1}+u_{m t},
\end{aligned}
$$


where

$$
\begin{aligned}
& \omega_{m}^{*}=\omega_{m}+\left(1-\sum_{j=1}^{q} \beta_{m m \cdot j}\right) E\left(\ln \eta_{m t}^{2}\right), \quad \boldsymbol{\phi}_{m . i}=\boldsymbol{\alpha}_{m . i}+\boldsymbol{\beta}_{m . i}, \\
& \theta_{m m \cdot j}=-\beta_{m m \cdot j} \quad \text { and } \quad u_{m t}=\ln \eta_{m t}^{2}-E\left(\ln \eta_{m t}^{2}\right) .
\end{aligned}
$$

Under stationarity and suitable regularity conditions, (21) can be estimated consistently with standard software. Subsequently, the log-GARCH parameters can be identified via the relations in (22). To identify $\omega_{m}$, the formula in (9) can be applied to the residuals of equation $m$ to estimate $E\left(\ln \eta_{m t}^{2}\right)$. For inference on the log-GARCH parameters in equation $m$, an approach similar to the one outlined in Section 2.4 can be used. For inference that involves parameters from more than one equation, then the joint coefficient covariance is needed, see Francq and Sucarrat (2017).

For equation-by-equation estimation to be available the GARCH-matrices $\boldsymbol{\beta}_{1}, \ldots, \boldsymbol{\beta}_{q}$ must all be diagonal. To test whether this is indeed the case, a Lagrange-Multiplier (LM) test of equation $m$ can be devised: Under the null all the elements of $\left\{\beta_{m i . j}: i \neq\right.$ $m, j=1, \ldots, q\}$ are equal to zero, whereas under the alternative one or more elements are non-zero. Formally, this has not been pursued yet in the theoretical log-GARCH literature.

\subsection{Non-stationary models}

A common approach to non-stationary volatility is to decompose $\boldsymbol{\sigma}_{t}^{2}$ multiplicatively, see (amongst other) Van Bellegem and Von Sachs (2004), Engle and Rangel (2008), Mazur and Pipien (2012), and Amado and Terasvirta (2014a, 2014b). This means

$$
\boldsymbol{\sigma}_{t}^{2}=\boldsymbol{g}_{t} \odot \boldsymbol{h}_{t}=\left(g_{1 t} h_{1 t}, \ldots, g_{M t} h_{M t}\right)^{\prime}
$$

where $\boldsymbol{g}_{t}$ is the non-stationary component, $\boldsymbol{h}_{t}$ is the stationary component (e.g. a GARCHlike process), and $\odot$ is the elementwise (Hadamard) matrix product. ${ }^{2}$ Escribano and $\mathrm{Su}-$ carrat (2018) propose a non-stationary multivariate log-GARCH-X specification that can be estimated equation-by-equation. Their motivation was the presence of non-stochastic periodicity in the intraday electricity price market. However, their idea applies more generally. The non-stationary component in their model is given by

$$
\ln \boldsymbol{g}_{t}=\left(\ln g_{1}\left(\boldsymbol{\lambda}_{1}^{f}, \boldsymbol{x}_{1 t}^{f}\right), \ldots, \ln g_{M}\left(\boldsymbol{\lambda}_{M}^{f}, \boldsymbol{x}_{M t}^{f}\right)\right)^{\prime}
$$

where $\ln g_{1}, \ldots, \ln g_{M}$ are known functions (linear or nonlinear), $\boldsymbol{x}_{1 t}^{f}, \ldots, \boldsymbol{x}_{M t}^{f}$ are known, non-stochastic or fixed (hence the superscript $f$ ) regressors, and $\boldsymbol{\lambda}_{1}^{f}, \ldots, \boldsymbol{\lambda}_{M}^{f}$ are unknown parameters to be estimated. Neither the $\boldsymbol{x}_{m t}^{f}$ 's nor the $\ln g_{m}$ 's are restricted to be equal across equations, and the $\ln g_{m}$ 's can assume a variety of shapes. In the simplest case the $\ln g_{m}$ 's are linear functions made up of time dummies (e.g. calendar effects), but it can also take the shape of an exponential spline as in Engle and Rangel (2008), the Fourier Flexible Form (FFF) as in Mazur and Pipien (2012), or smooth threshold models as in Amado

\footnotetext{
${ }^{2}$ For example, if $\boldsymbol{a}$ and $\boldsymbol{b}$ are two equally sized $M \times 1$ vectors, say, $\boldsymbol{a}=\left(a_{1}, \ldots, a_{M}\right)^{\prime}$ and $\boldsymbol{b}=$ $\left(b_{1}, \ldots, b_{M}\right)^{\prime}$, then $\boldsymbol{a} \odot \boldsymbol{b}=\left(a_{1} b_{1}, \ldots, a_{M} b_{M}\right)^{\prime}$.
} 
and Terasvirta (2014a, 2014b). The functions may also be estimated nonparametrically, as in Van Bellegem and Von Sachs (2004).

If we for notational simplicity exclude asymmetry and covariates, then the stationary component is given by

$$
\ln \boldsymbol{h}_{t}=\boldsymbol{\omega}+\sum_{i=1}^{p} \boldsymbol{\alpha}_{i} \ln \widetilde{\boldsymbol{\epsilon}}_{t-i}^{2}+\sum_{j=1}^{q} \boldsymbol{\beta}_{j} \ln \boldsymbol{h}_{t-j}
$$

where $\ln \boldsymbol{h}_{t}=\ln \boldsymbol{\sigma}_{t}^{2}-\ln \boldsymbol{g}_{t}=\left(\ln h_{1, t}, \ldots, \ln h_{M, t}\right)^{\prime}, \boldsymbol{\omega}=\left(\omega_{1}, \ldots, \omega_{M}\right)^{\prime}, \ln \widetilde{\boldsymbol{\epsilon}}_{t}^{2}=\left(\ln \boldsymbol{\epsilon}_{t}^{2}-\right.$ $\left.\ln \boldsymbol{g}_{t}\right)=\left(\ln h_{1 t} \eta_{1 t}^{2}, \ldots, \ln h_{M t} \eta_{M t}^{2}\right)^{\prime}$, and $\boldsymbol{\alpha}_{i}$ and $\boldsymbol{\beta}_{j}$ are both $M \times M$ matrices as in (15). The matrices $\boldsymbol{\beta}_{j}$ need not be diagonal. However, we will impose this restriction to enable an equation-by-equation estimation scheme. The $m$ th. log-volatility equation thus becomes

$$
\begin{aligned}
\ln \sigma_{m t}^{2} & =\ln g_{m t}+\ln h_{m t} \\
\ln g_{m t} & =\ln g_{m}\left(\boldsymbol{\lambda}_{m}^{f}, \boldsymbol{x}_{m t}^{f}\right) \\
\ln h_{m t} & =\omega_{m}+\sum_{i=1}^{p} \boldsymbol{\alpha}_{m . i} \ln \widetilde{\boldsymbol{\epsilon}}_{t-i}^{2}+\sum_{j=1}^{q} \beta_{m m . j} \ln h_{m, t-j}^{2},
\end{aligned}
$$

where $\boldsymbol{\alpha}_{m . i}$ is the $m$ th. row of $\boldsymbol{\alpha}_{i}$, i.e. $\boldsymbol{\alpha}_{m . i}=\left(\alpha_{m 1 . i}, \ldots, \alpha_{m M . i}\right)$. Let $\lambda_{m 0}^{f}$ denote the unconditional mean of $\ln \widetilde{\epsilon}_{m t}^{2}$, i.e. $\lambda_{m 0}^{f}=E\left(\ln \widetilde{\epsilon}_{m t}^{2}\right)$ with $E\left|\ln \widetilde{\epsilon}_{m t}^{2}\right|<\infty$. If we add $\ln \eta_{m t}^{2}$ to each side of (24), and then $\lambda_{m 0}^{f}-\lambda_{m 0}^{f}$ to the right-hand side, we obtain

$$
\ln \epsilon_{m t}^{2}=\lambda_{m 0}^{f}+\ln g_{m}\left(\boldsymbol{\lambda}_{m}^{f}, \boldsymbol{x}_{m t}^{f}\right)+w_{m t}, \quad w_{m t}=\left(\ln \widetilde{\epsilon}_{m t}^{2}-\lambda_{m 0}^{f}\right) .
$$

This is simply a regression with a fixed or non-stochastic part, i.e. $\lambda_{m 0}^{f}+\ln g_{m}\left(\boldsymbol{\lambda}_{m}^{f}, \boldsymbol{x}_{m t}^{f}\right)$, and a zero-mean stationary error governed by the mean-corrected ARMA model

$$
w_{m t}=\sum_{i=1}^{p} \boldsymbol{\phi}_{m . i} \boldsymbol{w}_{t-i}+\sum_{j=1}^{q} \theta_{m m . j} u_{m, t-j}+u_{m t}
$$

where $w_{m t}=\ln \widetilde{\epsilon}_{m t}^{2}-E\left(\ln \widetilde{\epsilon}_{m t}^{2}\right)$ and $\boldsymbol{w}_{t}=\left(w_{1 t}, \ldots, w_{M t}\right)^{\prime}$. This means the $m$ th. equation can be estimated in three steps:

1. Estimate $\lambda_{m 0}^{f}$ and $\boldsymbol{\lambda}_{m}^{f}$ via the auxiliary regression

$$
\ln \epsilon_{m t}^{2}=\lambda_{m 0}^{f}+\ln g_{m}\left(\boldsymbol{\lambda}_{m}^{f}, \boldsymbol{x}_{m t}^{f}\right)+w_{m t},
$$

where $\lambda_{m 0}$ is the intercept and $w_{m t}$ is a zero-mean stationary error-term governed by (27). If $\boldsymbol{\lambda}_{m}^{f}$ enters linearly in $\ln g_{m}$, then the parameters can simply be estimated by OLS.

2. Fit an ARMA model to the residuals $\widehat{w}_{m t}$ from the first step. The relation between the parameters of the log-GARCH model and the parameters of the mean-corrected ARMA-representation are the same as in the case where the ARMA-representation is not mean-corrected, i.e. (22). So this provides an estimate of all the log-GARCH parameters apart from the intercept $\omega_{m}$. An estimate of $\omega_{m}$, however, is not needed if the aim is to estimate $\sigma_{m t}^{2}$. The reason for this is that the fitted values from the 
first two steps provide estimates of $E\left(\ln \widetilde{\epsilon}_{m t}^{2}\right)+\ln g_{m t}$ and $E_{t-1}\left(y_{m t}\right)$, respectively. Adding these gives

$$
\begin{aligned}
E\left(\ln \widetilde{\epsilon}_{m t}^{2}\right)+\ln g_{m t}+E_{t-1}\left(w_{m t}\right) & =\ln g_{m t}+E_{t-1}\left(\ln \widetilde{\epsilon}_{m t}^{2}\right) \\
& =\ln g_{m t}+\ln h_{t}+E\left(\ln \eta_{m t}^{2}\right)
\end{aligned}
$$

since $\ln \widetilde{\epsilon}_{m t}^{2}=\ln h_{m t}+\ln \eta_{m t}^{2}$. So only an estimate of $E\left(\ln \eta_{m t}^{2}\right)$ is needed to complete the estimate of $\sigma_{m t}^{2}$.

3. Estimate the log-moment $E\left(\ln \eta_{m t}^{2}\right)$ needed to complete the estimate of $\sigma_{m t}^{2}$. Again, we can use the residuals from Step 2 in combination with (9).

Summarised, then, the estimate of $\sigma_{m t}^{2}$ is given by

$$
\widehat{\sigma}_{m t}^{2}=\exp (\underbrace{\widehat{E}\left(\ln \widetilde{\epsilon}_{m t}^{2}\right)+\ln \widehat{g}_{m t}}_{\text {Step } 1}+\underbrace{\widehat{E_{t-1}\left(w_{m t}\right)}}_{\text {Step 2 }}-\underbrace{\widehat{E}\left(\ln \eta_{m t}^{2}\right)}_{\text {Step 3 }})
$$

where $\widehat{E}\left(\ln \widetilde{\epsilon}_{m t}^{2}\right)+\ln \widehat{g}_{m t}$ is the fitted value of the auxiliary regression in Step 1, $\widehat{E}_{t-1}\left(y_{m t}\right)$ is the fitted value of the mean-corrected ARMA representation in Step 2, and $\widehat{E}\left(\ln \eta_{m t}^{2}\right)$ is the estimate of $E\left(\ln \eta_{m t}^{2}\right)$ in Step 3. Note that the three-step procedure can in fact be reduced to two steps if the centred exponential Chi-squared QMLE of Francq and Sucarrat (2018) is used in the second step, since $E\left(\ln \eta_{m t}^{2}\right)$ enters explicitly as a parameter to be estimated in the centred exponential Chi-squared density. This will also be more efficient if $\eta_{m t}$ is normal or close to normal.

An estimate of $\omega_{m}$ requires estimation of the other equations, in addition to equation $m$. This is because the expression for $E\left(\ln \widetilde{\epsilon}_{m t}^{2}\right)$, which can be written as $E\left(\ln \widetilde{\epsilon}_{m t}^{2}\right)=$ $\omega_{m}^{*}+\sum_{i=1}^{p} \phi_{m . i} E\left(\ln \widetilde{\boldsymbol{\epsilon}}_{t}^{2}\right)$, depends on the unconditional expectations of the other equations. Recalling, from (22), that $\omega_{m}^{*}=\omega_{m}+\left(1-\sum_{j=1}^{q} \beta_{m m . j}\right) E\left(\ln \eta_{m t}^{2}\right)$ when the GARCHmatrices are diagonal, solving for $\omega_{m}$ in the expression for $E\left(\ln \widetilde{\epsilon}_{m t}^{2}\right)$ gives

$$
\omega_{m}=\left(1-\sum_{j=1}^{q} \beta_{m m . j}\right) E\left(\ln \widetilde{\epsilon}_{m t}^{2}\right)-\sum_{i=1}^{p} \boldsymbol{\alpha}_{m . i} E\left(\ln \widetilde{\boldsymbol{\epsilon}}_{t}^{2}\right)-\left(1-\sum_{j=1}^{q} \beta_{m m . j}\right) E\left(\ln \eta_{m t}^{2}\right)
$$

where we have used that $\sum_{i=1}^{p} \boldsymbol{\phi}_{m . i} E\left(\ln \widetilde{\boldsymbol{\epsilon}}_{t}^{2}\right)=\sum_{i=1}^{p} \boldsymbol{\alpha}_{m . i} E\left(\ln \widetilde{\boldsymbol{\epsilon}}_{t}^{2}\right)+\sum_{j=1}^{q} \beta_{m m . j} E\left(\ln \widetilde{\epsilon}_{m t}^{2}\right)$. It should be noted that only the elements in $E\left(\ln \widetilde{\boldsymbol{\epsilon}}_{t}^{2}\right)$, apart from the $m$ th. entry, comes from the other equations. In other words, if there is no feedback effects (i.e. all entries in the $\boldsymbol{\alpha}_{m . i}$ 's apart from the $m$ th. entry are zero), then there is no need to estimate the other equations in order to estimate $\omega_{m}$.

Asymmetry and stochastic covariates ("X") can be added without affecting the estimation procedure just sketched. The only caveat is that they need to be mean corrected. Specifically, if $\boldsymbol{x}_{t-1}$ is a $(r+s) \times 1$ vector that collects all the asymmetry terms and conditioning covariates of the stationary part, then they need to enter as $\left(\boldsymbol{x}_{t-1}-\overline{\boldsymbol{x}}\right)$ in the ARMA representation, where $\overline{\boldsymbol{x}}=\left(\bar{x}_{1}, \ldots, \bar{x}_{M}\right)^{\prime}$ are the sample means of the stationary covariates. The stationary component is thus

$$
\ln \boldsymbol{h}_{t}^{2}=\boldsymbol{\omega}+\sum_{i=1}^{p} \boldsymbol{\alpha}_{i} \ln \widetilde{\boldsymbol{\epsilon}}_{t-i}^{2}+\sum_{j=1}^{q} \boldsymbol{\beta}_{j} \ln \boldsymbol{h}_{t-j}^{2}+\boldsymbol{\delta}\left(\boldsymbol{x}_{t-1}-\overline{\boldsymbol{x}}\right)
$$


where $\boldsymbol{\delta}$ is a parameter-matrix of appropriate size, and the mean-corrected ARMA representation of equation $m$ is

$$
w_{m t}=\sum_{i=1}^{p} \boldsymbol{\phi}_{m . i} \boldsymbol{w}_{t-i}+\sum_{j=1}^{q} \theta_{m m . j} u_{m, t-j}+\boldsymbol{\delta}_{m}\left(\boldsymbol{x}_{t-1}-\overline{\boldsymbol{x}}\right)+u_{m t},
$$

where $w_{m t}, \boldsymbol{w}_{t}$ and $u_{m t}$ are defined as earlier, and $\boldsymbol{\delta}_{m}$ is the $m$ th. row of $\boldsymbol{\delta}$. The practical consequence of this is that the three step estimation procedure described above only requires one minor modification: Estimate (29) instead of (27) in Step 2. The other steps are unchanged, and if an estimate of $\omega_{m}$ is needed, then formula (28) can still be used.

The asymptotic theory of non-stationary log-GARCH models has not been formally developed yet. Nevertheless, approximate inference procedures are readily available. For the stationary ARMA-representation a procedure similar to the one outlined in Section 2.4 can be used for inference within a single equation. The unknown is whether, or to what extent, this procedure is affected by the prior estimation of the non-stationary part. For inference that involves parameters from more than one equation, then an approximate joint coefficient covariance can be obtained along the lines of Francq and Sucarrat (2017). For inference regarding the parameters in the non-stationary part, then an approximate coefficient covariance can be computed by classical methods. For example, if the parameters of the non-stationary part in equation $m$ are estimated by OLS, and if $\boldsymbol{X}_{m}$ denotes the $T \times k$ regressor matrix of the OLS estimator, then an approximate expression is obtained as

$$
\left(\boldsymbol{X}_{m}^{\prime} \boldsymbol{X}_{m}\right)^{-1} \boldsymbol{X}_{m}^{\prime} \widehat{\Omega}_{m} \boldsymbol{X}_{m}\left(\boldsymbol{X}_{m}^{\prime} \boldsymbol{X}_{m}\right)^{-1}
$$

where $\widehat{\boldsymbol{\Omega}}_{m}$ is an estimate of the autocovariance matrix of $w_{m 1}, \ldots, w_{m T}$. The estimation results of the stationary part can be used to compute $\widehat{\Omega}_{m}$. Indeed, if the stationary part is an ARMA, then this procedure is already available in a number of softwares.

\subsection{Dynamic Conditional Correlations (DCCs)}

Assumption (19) allow for certain types of DCCs when a multivariate log-GARCH is estimated equation-by-equation. The estimation procedures described above, however, do not provide estimates of the DCCs. Nevertheless, they can - if needed - be estimated in a subsequent step. The estimates $\widehat{\sigma}_{1 t}^{2}, \ldots, \widehat{\sigma}_{M t}^{2}$ lead to the standardised residuals $\widehat{\boldsymbol{\eta}}_{t}=\left(\widehat{\eta}_{1 t}, \ldots, \widehat{\eta}_{M t}\right)^{\prime}$, where $\widehat{\eta}_{m t}=\epsilon_{m t} / \widehat{\sigma}_{m t}$. These residuals can be used to estimate a DCC specification of $\boldsymbol{R}_{t}=E\left(\boldsymbol{\eta}_{t} \boldsymbol{\eta}_{t}^{\prime} \mid \mathcal{F}_{t-1}\right)$. An example is the DCC of Engle (2002), or alternatively the corrected version of Aielli (2013), see e.g. the empirical section of Francq and Sucarrat (2017). Another option is the robust (to spikes) DCC model proposed for electricity prices by Dupuis (2017).

\section{Handling zeros in practice}

Throughout we have relied on the theoretical assumption $\operatorname{Pr}\left(\eta_{t}=0\right)=0$. In practice, however, if no conditional mean equation is fitted (i.e. we set $\mu_{t}=0$ for all $t$ ), we may experience that $\epsilon_{t}=0$ for some $t$. The most straightforward solution to this is to fit a specification $\mu_{t}$, e.g. an intercept. This is not only justifiable in most contexts, it is also recommendable in order to ensure that the fitted values of $\epsilon_{t}$ are centred about zero. 
A second solution consists of replacing zeros with some non-zero value $c$. One such value is a number very close to zero, say, the machine epsilon (e.g. 2.22e - 16) of the software used. This is probably the worst possible choice! The reason for this is that $\ln c^{2}$ will usually be much smaller than any empirical non-zero value of $\ln \epsilon_{t}^{2}$. Accordingly, this will induce a large ARCH shock (or inlier) at each zero location. A more sensible solution is to set $c$ equal to a value informed by the economic application in question. If there is no (obvious) economic motivation to inform the choice of $c$, then one may choose a certain quantile of the non-zero values of $\epsilon_{t}^{2}$ (e.g. 10\%), or the sample average of $\ln \epsilon_{t}^{2}$ (zeros excluded), or simply the value 1 . The latter is very neat and justified in the $\log -\operatorname{GARCH}(1,1)$ when the estimates of $\alpha_{1}$ and $\beta_{1}$ are typical, i.e. about 0.05 and 0.9 , respectively. Setting $c=1$ thus means $\ln c^{2}=0$, so that all the weight (in predicting $\ln \sigma_{t}^{2}$ ) is shifted on to the GARCH term, i.e. $\ln \sigma_{t-1}^{2}$. If $\beta_{1}$ is large (e.g. about 0.9), then this is a very sensible solution.

A third solution consists of estimating the replacement value. This is the solution proposed by Sucarrat and Escribano (2018). Specifically, they propose to treat zeros as missing values, and to impute the missing values by the estimate of $E_{t-1}\left(\ln \epsilon_{t}^{2}\right)$ at each missing location. This means an optimal replacement value is inserted at each missing location in the ARMA representation, where "optimal" means the conditional (squared) forecast error is minimised, and/or that the likelihood is maximised. Arguably, treating zeros as missing values is the most appealing solution if no conditional mean is fitted. However, implementing the solution usually requires more of the user, and consistent parameter estimates are not guaranteed - in particular if the proportion of zeros is large. The freely available $R$ package lgarch (Sucarrat (2015)) implements the missing value approach.

A fourth solution consists of adding a non-zero value $c$ to all the squared observations $\epsilon_{1}^{2}, \ldots, \epsilon_{T}^{2}$. This leads to a new series $\left\{\epsilon_{t}^{* 2}\right\}$ with $\epsilon_{t}^{* 2}=\epsilon_{t}^{2}+c$ and $\epsilon_{t}^{*}=\sigma_{t}^{*} \eta_{t}^{*}$, such that $\sigma_{t}^{* 2}$ is approximately equal to $\sigma_{t}^{2}+c$. In other words, approximate forecasts of $\sigma_{t}^{2}$ can be obtained by using the estimates of $\sigma_{t}^{* 2}$, and noting that $\sigma_{t}^{2} \approx \sigma_{t}^{* 2}-c$. If the values of $\epsilon_{t}^{2}$ are sufficiently large compared with $c$, then adding $c$ will not alter the dynamics of $\epsilon_{t}^{2}$ in a notable manner. An example is the case where $\epsilon_{t}^{2}$ is interpreted as volume, i.e. a positively valued variable (see Section 5 ). In this case $\epsilon_{t}^{2}$ will usually be much larger than, say, $c=1$.

\section{$5 \quad$ Modelling positively valued variables}

Engle and Russell (1998) noted that $\epsilon_{t}^{2}$ could be interpreted as positively valued variable, and hence showed that $\sigma_{t}^{2}$ can be interpreted as the conditional expectation of the positively valued variable. Put differently, Engle and Russell (1998) showed that the ARCH-class of models can be used to model positively valued variables like duration, volume, price-spread or realised volatility. This spurred the Multiplicative Error Model (MEM) literature, see Brownlees et al. (2012) for an overview. A particularly useful characteristic of the MEM interpretation is that, in practice, an $\mathrm{ARCH}$ estimation routine can be used to estimate a MEM. For example, suppose $y_{t}$ denotes the positively valued variable in question. By providing the software in question with $\sqrt{y}_{t}$, then the software will return estimates of the MEM. 
Formally, the MEM-class is given by

$$
y_{t}=\psi_{t} \zeta_{t}, \quad \zeta_{t} \sim i i d\left(1, \sigma_{\zeta}^{2}\right), \quad \psi_{t}, \zeta_{t} \geq 0
$$

where $\psi_{t}=E_{t-1}\left(y_{t}\right)$ is interpreted as the expectation of $y_{t}$ conditional on past information. Bauwens and Giot (2000) were the first to propose a logarithmic version of the MEM, i.e. a log-MEM. They noted that the MEM-interpretation associated with ordinary GARCH models was restricted by the non-negativity constraints on the parameters, and by the non-negativity constraints on the conditioning variables. Instead, they proposed a first order log-MEM with a single (stochastic) conditioning covariate. More generally, however, the univariate $\log \operatorname{MEM}(p, q)$ with stochastic conditioning variables can be written as

$$
\ln \psi_{t}=\omega+\sum_{i=1}^{p} \alpha_{i} \ln y_{t-i}+\sum_{j=1}^{q} \beta_{j} \ln \psi_{t-j}+\sum_{l=1}^{s} \lambda_{l} x_{l, t-1}
$$

That is, a univariate log-GARCH-X without asymmetry (ordinary and extended asymmetryterms are not meaningful in MEMs). If $\operatorname{Pr}\left(\zeta_{t}=0\right)=0$ and $E\left|\ln \zeta_{t}\right|<\infty$, then the model admits - as earlier - an ARMA-representation almost surely given by

$$
\ln y_{t}=\omega^{*}+\sum_{i=1}^{p} \phi_{i} \ln y_{t-i}+\sum_{j=1}^{q} \theta_{j} u_{t-j}+\sum_{l=1}^{s} \lambda_{l} x_{l, t-1}+u_{t}
$$

and the relations between the log-MEM and ARMA parameters are exactly the same as before, i.e. they are given by (22). The existence of an ARMA-representation of the logMEM was, to the best of my knowledge, first pointed out by Allen et al. (2008). Similarly, the model can be extended to the multivariate case as in Section 3, and non-stationarities can be introduced in the same way as in Section 3.3. Finally, fractionally integrated extensions of the log-MEM are considered in Beran et al. (2015), and in Feng and Zhou (2015).

\section{Conclusions}

The log-GARCH model provides a very flexible framework for the modelling of economic uncertainty, financial volatility and a range of other positively valued variables. In this chapter we have outlined how univariate and multivariate log-GARCH models can be represented by (V)ARMA-like representations, and - as a consequence - how well-known (V)ARMA results can be used to estimate univariate and multivariate log-GARCH models. Nevertheless, there is still a large, unexploited potential. There exists a wide range of well-known results on time-varying coefficients, non-stationarities, missing data and efficient estimation, amongst other, that can potentially be used to shed light on and further extend the log-GARCH class of models via its (V)ARMA-like representations.

\section{References}

Aielli, G. P. (2013). Dynamic Conditional Correlations: On Properties and Estimation. Journal of Business and Economic Statistics 31, 282-299. http://dx.doi.org/10. 1080/07350015.2013.771027. 
Allen, D., F. Chan, M. McAleer, and S. Peiris (2008). Finite sample properties of the QMLE for the log-acd model: Application to australian stocks. Journal of Econometrics $147,163-185$.

Amado, C. and T. Terasvirta (2014a). Modelling Changes in the unconditional variance of long stock return series. Journal of Empirical Finance 25, 15-35.

Amado, C. and T. Terasvirta (2014b). Modelling volatility by variance decomposition. Journal of Econometrics 175, 142-153.

Asai, M. (1998). A new method to estimate stochastic volatility models: A log-GARCH approach. Journal of the Japanese Statistical Society 28, 101-114.

Bauwens, L. and P. Giot (2000). The logarithmic ACD model: an application to the bid-ask quote process of three NYSE stocks. Annales d'Economie et de Statistique 60, 117-149.

Beran, J., Y. Feng, and S. Gosh (2015). Modelling long-range dependence and trends in duration series: an approach based on EFARIMA and ESEMIFAR models. Statistical Papers 56, 431-451.

Bollerslev, T. (1986). Generalized autoregressive conditional heteroscedasticity. Journal of Econometrics 31, 307-327.

Brockwell, P. J. and R. A. Davis (2006). Time Series: Theory and Methods. New York: Springer. 2nd. Edition, first published in 1991.

Brownlees, C., F. Cipollini, and G. Gallo (2012). Multiplicative Error Models. In L. Bauwens, C. Hafner, and S. Laurent (Eds.), Handbook of Volatility Models and Their Applications, pp. 223-247. New Jersey: Wiley.

Carnero, M. A., D. Pena, and E. Ruiz (2007). Effects of outliers on the identification and estimation of GARCH models. Journal of Time Series Analysis 28, 471-497.

Duan, N. (1983). Smearing Estimate: A Nonparametric Retransformation Method. Journal of the Americal Statistical Association 78, pp. 605-610.

Dupuis, D. J. (2017). Electricity price dependence in new york state zones: A robust detrended correlation approach. Annals of Applied Statistics 11, 248-273.

Engle, R. (1982). Autoregressive Conditional Heteroscedasticity with Estimates of the Variance of United Kingdom Inflations. Econometrica 50, 987-1008.

Engle, R. (2002). Dynamic Conditional Correlation: A Simple Class of Multivariate Generalized Autoregressive Conditional Heteroskedasticity Models. Journal of Business and Economic Statistics 20, 339-350.

Engle, R. F. and J. G. Rangel (2008). The Spline GARCH Model for Low Frequency Volatility and its Global Macroeconomic Causes. Review of Financial Studies 21, 11871222 . 
Engle, R. F. and J. R. Russell (1998). Autoregressive Conditional Duration: A New Model of Irregularly Spaced Transaction Data. Econometrica 66, 1127-1162.

Escribano, Á. and G. Sucarrat (2018). Equation-by-Equation Estimation of Multivariate Periodic Electricity Price Volatility. Energy Economics. In press. Working paper version: MPRA Paper No. 72736 at https://mpra.ub.uni-muenchen.de/72736/.

Feng, Y. and C. Zhou (2015). Forecasting financial market activity using a semiparametric fractionally integrated Log-ACD. International Journal of Forecasting 31, 349-363.

Francq, C. and G. Sucarrat (2017). An Equation-by-Equation Estimator of a Multivariate Log-GARCH-X Model of Financial Returns. Journal of Multivariate Analysis 153, 1632 .

Francq, C. and G. Sucarrat (2018). An Exponential Chi-Squared QMLE for Log-GARCH Models Via the ARMA Representation. Journal of Financial Econometrics 16, 129154. Working Paper version: http://mpra.ub.uni-muenchen.de/51783/.

Francq, C. and L. Q. Thieu (2018). Qml inference for volatility models with covariates. Econometric Theory. https://doi.org/10.1017/S0266466617000512.

Francq, C., O. Wintenberger, and J.-M. Zakoïan (2013). GARCH Models Without Positivity Constraints: Exponential or Log-GARCH? Journal of Econometrics 177, 34-36.

Francq, C., O. Wintenberger, and J.-M. Zakoïan (2017). Goodness-of-fit tests for $\log$ and exponential GARCH models. TEST. http://dx.doi.org/10.1007/ s11749-016-0506-2.

Francq, C. and J.-M. Zakoïan (2016). Estimating multivariate volatility models equation by equation. The Journal of the Royal Statistical Society. Series B 78, 613-635. Working paper version: MPRA Paper No. 54250: http://mpra.ub.uni-muenchen.de/54250/.

Geweke, J. (1986). Modelling the Persistence of Conditional Variance: A Comment. Econometric Reviews 5, 57-61.

Glosten, L. R., R. Jagannathan, and D. E. Runkle (1993). On the Relation between the Expected Value and the Volatility of the Nominal Excess Return on Stocks. Journal of Finance 48, 1779-1801.

Harvey, A. C. and G. Sucarrat (2014). EGARCH models with fat tails, skewness and leverage. Computational Statistics and Data Analysis 76, 320-338.

Ljung, G. and G. Box (1979). On a Measure of Lack of Fit in Time Series Models. Biometrika 66, 265-270.

Mazur, B. and M. Pipien (2012). On the Empirical Importance of Periodicity in the Volatility of Financial Returns - Time Varying GARCH as a Second Order APC(2) Process. Central European Journal of Economic Modelling and Econometrics 4, 95-116.

Milhøj, A. (1987). A Multiplicative Parametrization of ARCH Models. Research Report 101, University of Copenhagen: Institute of Statistics. 
Nelson, D. B. (1991). Conditional Heteroskedasticity in Asset Returns: A New Approach. Econometrica 59, 347-370.

Pantula, S. (1986). Modelling the Persistence of Conditional Variance: A Comment. Econometric Reviews 5, 71-73.

Park, R. (1966). Estimation with Heteroscedastic Error Terms. Econometrica 34, 888888.

Pretis, F., J. Reade, and G. Sucarrat (2018). Automated General-to-Specific (GETS) Regression Modeling and Indicator Saturation for Outliers and Structural Breaks. Journal of Statistical Software 86, 1-44.

Psaradakis, Z. and E. Tzavalis (1999). On regression-based tests for persistence in logarithmic volatility models. Econometric Reviews 18, 441-448.

R Core Team (2014). R: A Language and Environment for Statistical Computing. Vienna, Austria: R Foundation for Statistical Computing.

R Core Team (2018). R: A Language and Environment for Statistical Computing. Vienna, Austria: R Foundation for Statistical Computing.

Sucarrat, G. (2015). lgarch: Simulation and Estimation of Log-GARCH Models. R package version $0.6-2$.

Sucarrat, G. and Á. Escribano (2018). Estimation of Log-GARCH Models in the Presence of Zero Returns. European Journal of Finance 24, 809-827. http://dx.doi.org/10. 1080/1351847X.2017.1336452.

Sucarrat, G., S. Grønneberg, and Á. Escribano (2016). Estimation and Inference in Univariate and Multivariate Log-GARCH-X Models When the Conditional Density is Unknown. Computational Statistics and Data Analysis 100, 582-594.

Trapletti, A. and K. Hornik (2016). tseries: Time Series Analysis and Computational Finance. R package version $0.10-35$.

Van Bellegem, S. and R. Von Sachs (2004). Forecasting economic time-series with unconditional time-varying variance. International Journal of Forecasting 20, 611-627. 
Table 1: Empirical examples of log-GARCH models (see Section 2.5)

\begin{tabular}{|c|c|c|c|c|c|}
\hline \multicolumn{6}{|c|}{ Descriptive statistics: } \\
\hline & Sample & $T$ & $s^{2}$ & $s^{4}$ & $\underset{[p-\text { val }]}{\mathrm{ARCH}}$ \\
\hline FTSE100 & $5 / 1 / 1998-2 / 6 / 2015$ & 4397 & 1.51 & 8.59 & $\begin{array}{l}97.9 \\
{[0.00]}\end{array}$ \\
\hline SP100 & $5 / 1 / 1998-1 / 6 / 2015$ & 4379 & 1.60 & 10.10 & $\begin{array}{l}220.1 \\
{[0.00]}\end{array}$ \\
\hline Apple & $10 / 9 / 1984-12 / 10 / 2011$ & 6835 & 9.63 & 53.85 & $\begin{array}{l}5.90 \\
{[0.02]}\end{array}$ \\
\hline USD/EUR & $5 / 1 / 1999-12 / 10 / 2011$ & 3274 & 0.45 & 5.45 & $\begin{array}{l}205.9 \\
{[0.00]}\end{array}$ \\
\hline Oilprice & $21 / 5 / 1987-4 / 10 / 2011$ & 6190 & 5.59 & 17.47 & $\begin{array}{l}78.6 \\
{[0.00]}\end{array}$ \\
\hline Gold & $4 / 1 / 2006-12 / 10 / 2011$ & 1448 & 1.96 & 6.18 & $\begin{array}{l}6.76 \\
{[0.01]}\end{array}$ \\
\hline
\end{tabular}

Plain log-GARCH(1,1) models:

\begin{tabular}{|c|c|c|c|c|}
\hline & $\widehat{\omega}$ & $\begin{array}{c}\widehat{\alpha}_{1} \\
\text { (s.e.) }\end{array}$ & $\begin{array}{c}\widehat{\beta}_{1} \\
\text { (s.e.) }\end{array}$ & $\widehat{E}\left(\ln \eta_{t}^{2}\right)$ \\
\hline FTSE100 & 0.067 & $\begin{array}{l}0.047 \\
(0.006)\end{array}$ & $\begin{array}{l}0.942 \\
(0.008)\end{array}$ & -1.415 \\
\hline SP100 & 0.072 & $\begin{array}{l}0.047 \\
(0.006)\end{array}$ & $\begin{array}{l}0.945 \\
(0.008)\end{array}$ & -1.513 \\
\hline Apple & 0.055 & $\begin{array}{c}0.032 \\
(0.005)\end{array}$ & $\begin{array}{l}0.963 \\
(0.007)\end{array}$ & -1.375 \\
\hline USD/EUR & 0.025 & $\begin{array}{l}0.022 \\
(0.005)\end{array}$ & $\begin{array}{l}0.971 \\
(0.007)\end{array}$ & -1.380 \\
\hline Oilprice & 0.069 & $\begin{array}{l}0.037 \\
(0.005)\end{array}$ & $\begin{array}{l}0.952 \\
(0.007)\end{array}$ & -1.401 \\
\hline Gold & 0.054 & $\begin{array}{c}0.032 \\
(0.007)\end{array}$ & $\begin{array}{l}0.958 \\
(0.010)\end{array}$ & -1.522 \\
\hline
\end{tabular}

Asymmetric log-GARCH(1,1) models:

\begin{tabular}{|c|c|c|c|c|c|c|}
\hline & $\widehat{\omega}$ & $\begin{array}{c}\widehat{\alpha}_{1} \\
(\text { s.e. })\end{array}$ & $\begin{array}{c}\widehat{\beta}_{1} \\
\text { (s.e.) }\end{array}$ & $\begin{array}{c}\widehat{\gamma}_{1} \\
(\text { s.e. })\end{array}$ & $\begin{array}{c}\widehat{\lambda}_{1} \\
\text { (s.e.) }\end{array}$ & $\widehat{E}\left(\ln \eta_{t}^{2}\right)$ \\
\hline FTSE100 & -0.126 & $\begin{array}{l}0.003 \\
(0.006)\end{array}$ & $\begin{array}{l}0.945 \\
(0.006)\end{array}$ & $\begin{array}{c}0.069 \\
(0.010)\end{array}$ & $\begin{array}{c}0.362 \\
(0.035)\end{array}$ & -1.365 \\
\hline SP100 & -0.112 & $\begin{array}{l}0.011 \\
(0.007)\end{array}$ & $\begin{array}{c}0.934 \\
(0.008)\end{array}$ & $\begin{array}{l}0.070 \\
(0.012)\end{array}$ & $\begin{array}{c}0.374 \\
(0.040)\end{array}$ & -1.453 \\
\hline Apple & 0.046 & $\begin{array}{l}0.028 \\
(0.007)\end{array}$ & $\begin{array}{l}0.954 \\
(0.009)\end{array}$ & $\begin{array}{l}0.016 \\
(0.007)\end{array}$ & $\begin{array}{l}0.053 \\
(0.019)\end{array}$ & -1.361 \\
\hline USD/EUR & 0.013 & $\begin{array}{l}0.024 \\
(0.007)\end{array}$ & $\begin{array}{l}0.970 \\
(0.007)\end{array}$ & $\begin{array}{c}-0.003 \\
(0.010)\end{array}$ & $\begin{array}{l}0.021 \\
(0.027)\end{array}$ & -1.374 \\
\hline Oilprice & 0.058 & $\begin{array}{l}0.030 \\
(0.006)\end{array}$ & $\begin{array}{l}0.952 \\
(0.007)\end{array}$ & $\begin{array}{l}0.014 \\
(0.008)\end{array}$ & $\begin{array}{l}0.024 \\
(0.016)\end{array}$ & -1.396 \\
\hline Gold & 0.063 & $\begin{array}{l}0.036 \\
(0.009) \\
\end{array}$ & $\begin{array}{c}0.961 \\
(0.011)\end{array}$ & $\begin{array}{c}-0.012 \\
(0.014)\end{array}$ & $\begin{array}{c}-0.025 \\
(0.049) \\
\end{array}$ & -1.517 \\
\hline
\end{tabular}

$T$, number of non-missing returns. $s^{2}$, sample variance. $s^{4}$, sample kurtosis. ARCH, Ljung and Box (1979) test statistic of first-order serial correlation in the squared return. $p$-val, the $p$-value of the test-statistic. s.e., approximate standard errors (obtained via the numerically estimated Hessian) of estimate. All computations in $R$ (R Core Team (2014)), estimation with the lgarch function (Sucarrat (2015)). 

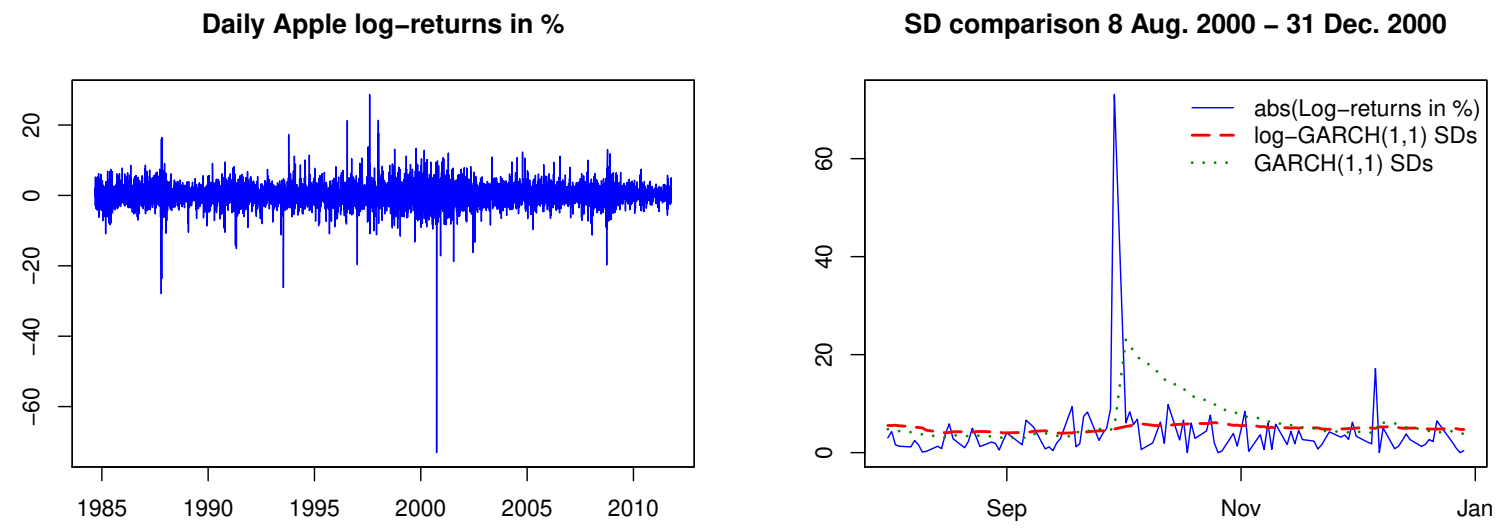

Figure 1: Daily Apple log-return in \% (left graph) and a snapshot (right graph) of the period before and after a profit warning 28 September 2000, see Section 1. Datasource: Yahoo Finance 



Figure 2: Daily financial log-returns in \%, see Section 2.5 and Table 1. Datasources: Bloomberg, Yahoo Finance, European Central Bank, US Energy Information Agency and Kitco 